\title{
Austrostipa, a new genus, and new names for Australasian species formerly included in Stipa (Gramineae)
}

\author{
S.W.L. Jacobs and J. Everett
}

\begin{abstract}
Jacobs, S.W.L., and Everett, J. (Royal Botanic Gardens, Sydney, Australia, 2000) 1996. Austrostipa, a new genus, and new names for species in Australasia formerly included in Stipa (Gramineae). Telopea 6(4): 579-595. The genus Austrostipa is described to include all of the native Australian species formerly included in Stipa, all relevant new combinations are provided, and a new species, Austrostipa geoffreyi, is described. Austrostipa is divided into 13 subgenera. The generic placements of the species introduced to Australia in either Nassella or Achnatherum are confirmed, new combinations are provided for the New Zealand Achnatherum petriei and the introduced Achnatherum caudatum, and the genus Anemanthele is retained. Keys are provided to genera of the Stipeae and to the subgenera of Austrostipa.
\end{abstract}

\section{Introduction}

The tribe Stipeae has been defined in several ways (Clifford \& Watson 1977, Clayton \& Renvoize 1986) relying on anatomical, micromorphological and floral characters. The shared derived character that so far best defines the tribe is the Stipoid embryo, a modified Pooid embryo that is small relative to the endosperm, has (i) the scutellum and embryonic leaf traces diverging from the same point with no internode, (ii) an epiblast, (iii) the scutellum and coleorrhiza fused, (iv) the embryonic leaf margins not overlapping, and (v) the primary root bent at a sharp angle from the main axis of the embryo (Reeder 1957). Correlated with this, but not exclusive to the Stipeae, are single-flowered spikelets, disarticulation above the glumes, and absence of a rhachilla extension. Current work on rDNA (ITS) in the Stipeae is confirming that the tribe is monophyletic (Hsiao et al. 1995, Hsiao pers. comm.).

We (Vickery et al. 1986, Jacobs et al. 1989) suggested that 61 endemic Australian, one endemic New Zealand and five introduced species were congeneric with Stipa L. but that a broader study would be needed to clarify the relationships. To date we recognise one species of Anemanthele endemic to New Zealand, one introduced species of Nassella (N. trichotoma) and one introduced species of Piptatherum (P. miliaceum) (Jacobs \& Everett 1993).

Since our statements on the generic relationships Barkworth \& Everett (1987), Everett (1990) and Barkworth $(1990,1993)$ have presented results from cladistic analyses of the species and genera of the tribe. Hsiao (pers. comm.) and Hsiao et al. (1995) have also been analysing nuclear rDNA (ITS) sequences. As a result of the earlier studies Barkworth (1990) made 68 new combinations in Nassella, and (Barkworth 1993) 36 new combinations and one new species in Achnatherum, and five new combinations in Hesperostipa. Barkworth (1993) recognised nine genera in the 'core' or traditional part of the tribe Stipeae, Achnatherum, Piptatherum, Oryzopsis, Ptilagrostis, Piptochaetium, Nassella, Hesperostipa, Stipa and Anemanthele. She also acknowledged that the Australian species may not fit into any of these genera. 
Everett (1990) analysed 30 morphological and anatomical characters from 37 taxa. The results are summarised in Fig. 1 being the strict consensus tree from PAUP 2.4 (Swofford 1985). The taxa are: Achnatherum, 'Boreobtusae' and 'Obtusae' (Barkworth \& Everett 1987), Nassella, Oryzopsis, Piptatherum sections Piptatherum, Miliacea (included with Piptatherum in Fig. 1) and Virescentia, Piptochaetium, Ptilagrostis, Hesperostipa, Stipa s.s., Stipa sections Podopogon (included in Piptochaetium in Fig. 1) and Stephanostipa (included in Nassella in Fig. 1), and 23 groups obtained from an analysis of Australian taxa (Everett 1990). Nardus and Joinvillea were used as outgroups. The 23 Australian groups were obtained by grouping the species into minimum unequivocal monophyletic units. This confirmed that the Australian species represented a monophyletic group (Austrostipa in Fig. 1), a sister group to Achnatherum and Ptilagrostis (Fig. 1), supporting a similar more tentative conclusion reached by Barkworth and Everett (1987). The rDNA study so far is indicating that the Australian species are more closely related to Nassella than to Achnatherum (Hsiao pers. comm.). If segregate genera are to be recognised then it is evident that the Australian species can no longer remain in Stipa s.s. Given that there is some conflict as to the closest relatives of the Australian species, and that all studies to date indicate that they are all more closely related to each other than any are to any non-Australian species, the best option is to place them in a new genus, here described as Austrostipa.

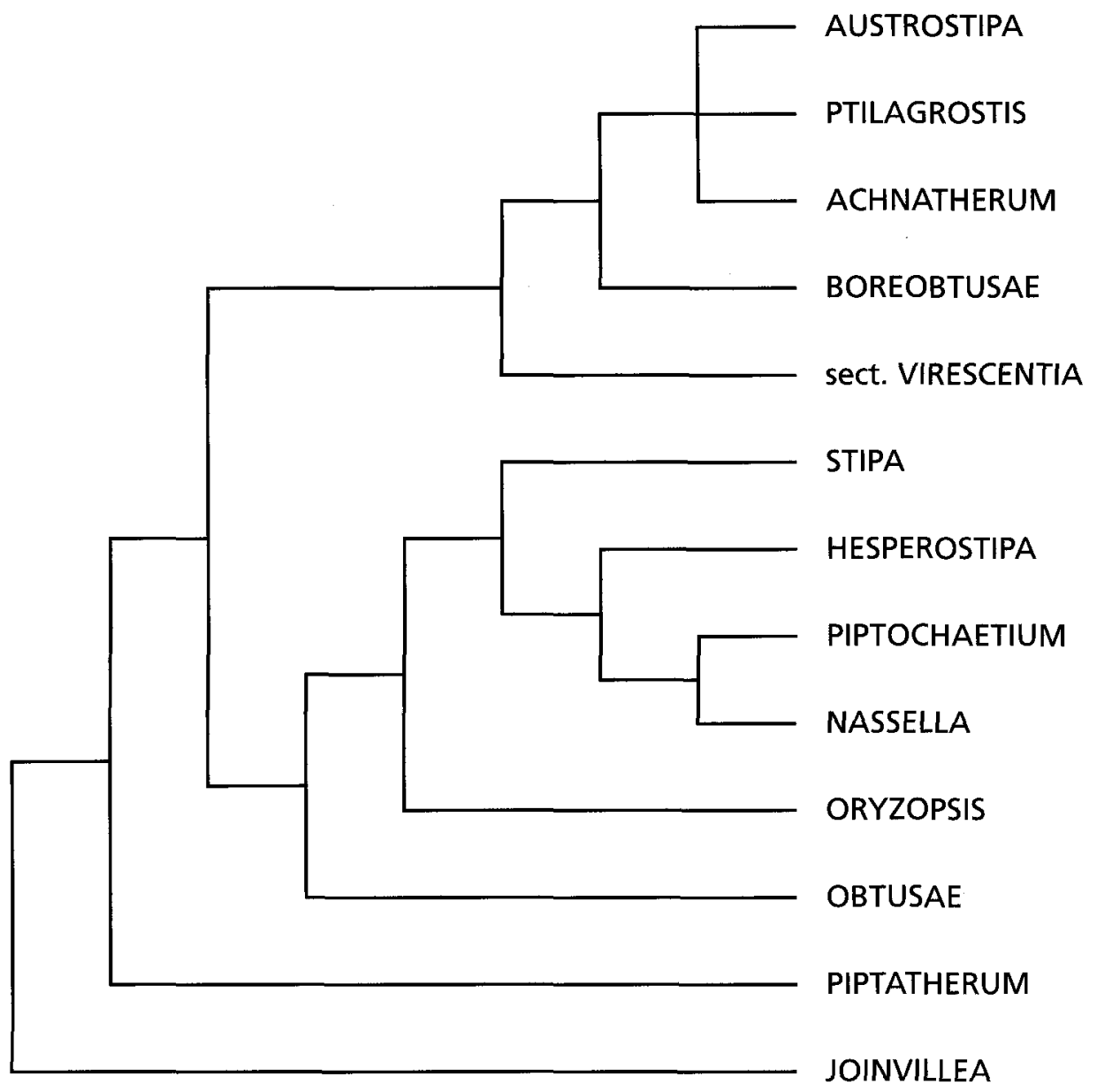

Fig. 1. A cladogram indicating relationships in the Stipeae, based on morphological and anatomical data, simplified (several groups removed) and redrawn from the consensus tree in Everett (1990). 


\section{Key to genera (modified after Barkworth 1993).}

1 Stamen 1; hilum elliptic; lemma nerves 3 ....................................... Anemanthele

$1^{*}$ Stamens 3; hilum linear; lemma nerves 3 or more $\quad$............................................ 2

2 Palea longitudinally grooved, longer than the lemma and protruding from between the lemma margins at the lemma apex; lemma margins involute, fitting into the paleal groove; lemma epidermis with very long fundamental cells with thickened sidewalls such that the surface appears longitudinally finely ridged; silica cells absent from lemma epidermis

Piptochaetium

2 Palea flat, shorter than or equalling the lemma, lemma margins flat, rarely palea grooved and then lemma margins involute, but then palea scarcely protruding at the lemma apex (two native Australian spp.); lemma surface smooth or tuberculate, not longitudinally ridged; lemma epidermal silica cells present or absent

3 Silica bodies in lemma epidermis square or rectangular

Stipa s.s.

$3^{*}$ Silica bodies in lemma epidermis round, oval or absent

4 Palea $<30 \%$ lemma length; lemma heavily silicified, tough, margins tightly overlapping; fundamental cells of lemma epidermis very short, much shorter than wide

Nassella

4* Palea usually $>30 \%$ lemma length, rarely shorter; lemma membranous, leathery or sometimes tough, the margins open, loosely or more or less tightly overlapping; fundamental cells of lemma epidermis usually much longer than wide

5 Palea coriaceous, not indurate; lemma pale, the margins scarcely enclosing the palea; floret terete or slightly dorsally compressed; callus very short and blunt; awn weak

$5^{*}$ Palea indurate; lemma pale or dark; lemma margins enclosing the palea; callus often pungent; awn robust

6 Leaf blades filiform, $<0.5 \mathrm{~mm}$ wide; plants of boggy alpine and subalpine habitats Ptilagrostis

$6^{*}$ Leaf blades not filiform, usually $>0.5 \mathrm{~mm}$ wide; plants of various habitats $\quad \ldots . . \quad 7$

7 Palea pubescent, shorter than or equal to the lemma but not prow-tipped

Achnatherum

$7^{*}$ Palea glabrous, prow-tipped, as long as the lemma

8 Florets dorsally compressed; lemma margins not overlapping, the palea exposed, at least in part

Piptatherum

8* Florets terete or laterally compressed; lemma margins often overlapping, concealing the palea

Oryzopsis

9 Lemma white, yellow, brown, red-brown, purple or black at maturity, often with brownish hairs; florets 2-12 mm long; awn 1.4-20 cm long; lemma epidermis always with round to oval silica bodies

Austrostipa

9* Lemma white or cream-coloured, with sparse white hairs; florets $>7.5 \mathrm{~mm}$ long; awn $>8 \mathrm{~cm}$ long; lemma epidermis without silica bodies

Hesperostipa

As well as the 62 Australian species of Austrostipa (including one new species described here) there are (Jacobs et al. 1995) three species of Achnatherum (one new combination 
provided below) introduced to Australia, five species of Nassella, and one species of Piptochaetium (P. montevidense) recorded from Victoria (Walsh 1994). After further examination there is still justification for maintaining Anemanthele, the stamen number of one (three in the rest of the tribe) reinforcing the other characters listed by Barkworth. \& Everett (1987). Anemanthele is more closely related to Achnatherum than to Austrostipa. Stipa petriei from New Zealand has the diagnostic characters of Achnatherum and is here included in that genus (new combination provided below).

\section{Achnatherum}

Achnatherum P. Beauvois (1812: 146).

LECTOTYPE (Niles and Chase 1925): Achnatherum calamagrostis (L.) P. Beauvois.

Achnatherum brachychaetum (Godr.) Barkworth (1993: 6).

Achnatherum caudatum (Trin.) S.W.L. Jacobs \& J. Everett, comb. nov.

Basionym: Stipa caudata Trinius, Mém. Acad. Imp.Sci.- St Pétersbourg Sér. 6, Sci. Math 1: 75 (1830); Vickery, Jacobs \& Everett (1986: 38-39).

Achnatherum papposum (Nees) Barkworth (1993:11).

Although currently included in Achnatherum, there is some doubt as to whether this and related species best belong here.

Achnatherum petriei (Buchanan) S.W.L. Jacobs \& J. Everett, comb. nov.

Basionym: Stipa petriei Buchanan, Indigenous Grasses N.Z. t. 17: 2 (1880); Jacobs et al. (1989).

\section{Austrostipa}

Vickery et al. (1986) recognised 10 informal groups in what is here described as Austrostipa, with a few species belonging to more than one group. Although Everett (1990) produced 23 unequivocal monophyletic groups, these can be rationalised to 13 groups (Fig. 2). Although some of these groups are not well supported by Everett's cladistic analyses, this is mainly because of poor resolution in parts of the cladogram. The cladogram produces a strong congruence with the groups suggested by Vickery et al. (1986), with some groups in that publication split (groups D, H and I), some fully supported (e.g. subgenera Tuberculatae and Petaurista of this paper, equivalent to groups $\mathrm{K}$ and $\mathrm{C}$ in Vickery et al. 1986) and others at least not contradicted if not supported (e.g. subgenera Austrostipa, Lobatae and Falcatae of this paper, equivalent to groups J, G and L in Vickery et al. 1986). We have recognised as subgenera our informal groups (Vickery et al. 1986) except where the cladograms (Everett 1990) supported such groups being split. The groups now split include subg. Bambusina being separated from what is here described as subg. Arbuscula (both previously group D), subg. Eremophilae being separated from what is here described as subg. Lancea (both previously group I), and subg. Aulax being separated from what is here described as subg. Ceres (both previously group $\mathrm{H}$ ).

The 13 groups we recognise in Austrostipa are here formally described as subgenera.

Austrostipa S.W.L. Jacobs \& J. Everett, gen. nov. Ex affinitate Achnatheri sed flosculis maturis fuscioribus tenacioribusque, marginis lemmatum plerumque imbricatis, callo grandiore, differt.

Caespitose or spreading, often rhizomatous facultative perennials. Leaves and branches either basal or cauline, sometimes forming intricate shrubby growth. Spikelets superficially all alike, 1-flowered, with the rhachilla not produced beyond the floret, hermaphrodite. Glumes persistent, hyaline to chartaceous, narrow, more 
or less keeled, often acute or acuminate, rarely muticous or mucronate, 1-5(-7)nerved, equal or unequal, usually longer than the floret (excluding awn). Floret disarticulating above the glumes, several times longer than wide, cylindrical, fusiform, pyriform or turbinate, rarely slightly gibbous, with a long, oblique, bearded, usually pungent-pointed (or rarely short and obtuse) callus. Lemma coriaceous, indurated, with convolute (or rarely involute) margins usually enclosing the palea and flower, 3-5(-7)-nerved, tapering at the tip and sometimes, though often only minutely, 1- or 2-lobed, awned from the tip or between the lobes. Awn flexuose or once or twice geniculate, with a twisted column (when mature) and a straight or curved bristle, variously glabrous to plumose. Palea membranous, hyaline or somewhat indurated, nerveless or 2-nerved, subequal to or shorter than the lemma. Lodicules 3 or 2, membranous, glabrous, lanceolate to spathulate, non-vascular. Stamens 3, frequently penicillate. Ovary glabrous; styles 2, free; stigmas plumose, tips exserted. Caryopsis

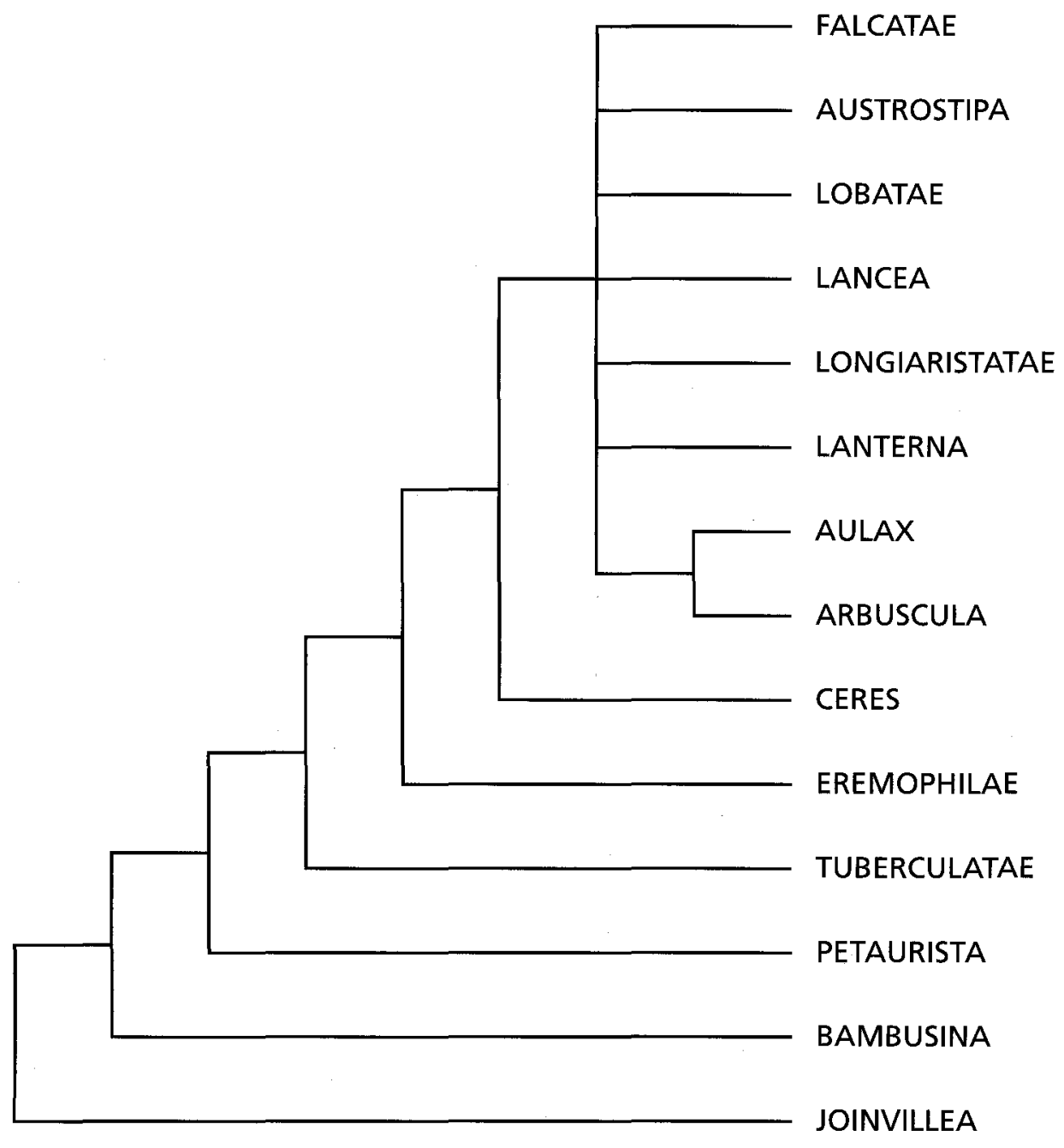

Fig. 2. Relationships of the subgenera of Austrostipa, based on a consensus cladogram from Everett (1990) but manually redrawn with some extra characters interpolated, some corrections to character scoring, and some simplification. Joinvillea is the outgroup. 
fusiform-terete, tightly enclosed by the lemma and palea; the embryo about 20-35\% the length of the grain; the hilum linear, nearly as long as the grain.

Type species: Austrostipa mollis (R. Br.) S.W.L. Jacobs \& J. Everett.

Austrostipa is closest to Achnatherum (Barkworth and Everett 1986; Everett 1990; Barkworth 1993) but differs from that genus in having the mature florets generally darker and tougher in texture, the lemma margins overlapping, and the callus longer, tougher and usually more pungent. Not all species of Austrostipa have all of these characteristics, but any one species has most of them. Austrostipa has very variable lemma epidermal characteristics.

Stipa s. str. is characterised by large (often $>1 \mathrm{~cm}$ long) chartaceous spikelets with a long (frequently $>10 \mathrm{~cm}$ long) plumose terminal awn and a comparatively short callus, and lemma epidermal silica bodies square, rectangular or, rarely, oval.

Austrostipa acrociliata (Reader) S.W.L. Jacobs \& J. Everett, comb. nov.

Basionym: Stipa acrociliata Reader, Victorian Naturalist 13: 167 (1897); Vickery, Jacobs \& Everett (1986: 25).

Austrostipa aphylla (Rodway) S.W.L. Jacobs \& J. Everett, comb. nov.

Basionym: Stipa pubescens var. aphylla Rodway, Tasmanian Fl.: 262 (1903).

Stipa aphylla (Rodway) Townrow (1970: 85); Vickery, Jacobs \& Everett (1986: 26-27).

Austrostipa aquarii (Vickery, S.W.L. Jacobs \& J. Everett) S.W.L. Jacobs \& J. Everett, comb. nov.

Basionym: Stipa aquarii Vickery, S.W.L. Jacobs \& J. Everett, Telopea 3: 27-28 (1986).

Austrostipa aristiglumis (F. Muell.) S.W.L. Jacobs $\mathcal{E}$ J. Everett, comb. nov.

Basionym: Stipa aristiglumis F. Mueller, Trans. \& Proc. Victorian Inst. Advancem. Sci.: 43 (1855); Vickery, Jacobs \& Everett (1986: 28-29).

Austrostipa bigeniculata (Hughes) S.W.L. Jacobs \& J. Everett, comb. nov.

Basionym: Stipa bigeniculata Hughes, Kew Bull. 1922: 20 (1922); Vickery, Jacobs \& Everett (1986: 30-31).

Austrostipa blackii (C.E. Hubb.) S.W.L. Jacobs \& J. Everett, comb. nov.

Basionym: Stipa blackii C.E. Hubbard, Kew Bull. 1925: 431 (1925); Vickery, Jacobs \& Everett (1986: 32-33).

Austrostipa blakei (Vickery, S.W.L. Jacobs \& J. Everett) S.W.L. Jacobs \& J. Everett, comb. nov.

Basionym: Stipa blakei Vickery, S.W.L. Jacobs \& J. Everett, Telopea 3: 34-35 (1986).

Austrostipa breviglumis (J.M. Black) S.W.L. Jacobs \& J. Everett, comb. nov.

Basionym: Stipa breviglumis J.M. Black, Trans. \& Proc. Roy. Soc. S. Austral. 65: 33 (1941); Vickery, Jacobs \& Everett (1986: 35-36).

Austrostipa campylachne (Nees) S.W.L. Jacobs\& J. Everett, comb. nov.

Basionym: Stipa campylachne Nees in Lehmann, Pl. Preiss. 2: 99 (1846); Vickery, Jacobs \& Everett (1986: 36-37).

Austrostipa centralis (Vickery, S.W.L. Jacobs \& J. Everett) S.W.L. Jacobs \& J. Everett, comb. nov.

Basionym: Stipa centralis Vickery, S.W.L. Jacobs \& J. Everett, Telopea 3: 39-40 (1986).

Austrostipa compressa (R. Br.) S.W.L. Jacobs \& J. Everett, comb. nov.

Basionym: Stipa compressa R. Brown, Prodr.: 175 (1810); Vickery, Jacobs \& Everett (1986: 40-41). 
Austrostipa crinita (Gaudich.) S.W.L. Jacobs \& J. Everett, comb. nov.

Basionym: Stipa crinita Gaudichaud in Freycinet, Voy. Uranie Bot.: 407 (1830); Vickery, Jacobs \& Everett (1986: 41-42).

Austrostipa curticoma (Vickery) S.W.L. Jacobs $\mathcal{E}$ J. Everett, comb. nov.

Basionym: Stipa curticoma Vickery, Telopea 2: 11 (1980); Vickery, Jacobs \& Everett (1986: 42-43).

Austrostipa densiflora (Hughes) S.W.L. Jacobs \& J. Everett, comb. nov.

Basionym: Stipa densiflora Hughes, Kew Bull. 1921: 18 (1921); Vickery, Jacobs \& Everett (1986: 44-45).

Austrostipa dongicola (Vickery, S.W.L. Jacobs \& J. Everett) S.W.L. Jacobs \& J. Everett, comb. nov.

Basionym: Stipa dongicola Vickery, S.W.L. Jacobs \& J. Everett, Telopea 3: 46 (1986).

Austrostipa drummondii (Steud.) S.W.L. Jacobs \& J. Everett, comb. nov.

Basionym: Stipa drummondii Steudel, Syn. Pl. Glum. 1: 128 (1854); Vickery, Jacobs \& Everett (1986: 47-48).

Austrostipa echinata (Vickery, S.W.L. Jacobs \& J. Everett) S.W.L. Jacobs \& J. Everett, comb. nov.

Basionym: Stipa echinata Vickery, S.W.L. Jacobs \& J. Everett, Telopea 3: 50 (1986).

Austrostipa elegantissima (Labill.) S.W.L. Jacobs $\mathcal{E}$ J. Everett, comb. nov.

Basionym: Stipa elegantissima Labillardiäre, Nov. Holl. Pl. 1: 23 (1804); Vickery, Jacobs \& Everett (1986: 51-53).

Austrostipa eremophila (Reader) S.W.L. Jacobs \& J. Everett, comb. nov.

Basionym: Stipa eremophila Reader, Victorian Naturalist 17: 154 (1901); Vickery, Jacobs \& Everett (1986: 53-55).

Austrostipa exilis (Vickery) S.W.L. Jacobs \& J. Everett, comb. nov.

Basionym: Stipa exilis Vickery, Telopea 2: 13 (1980); Vickery, Jacobs \& Everett (1986: 56-57).

Austrostipa feresetacea (Vickery, S.W.L. Jacobs $\mathcal{E}$ J. Everett) S.W.L. Jacobs $\mathcal{E}$ J. Everett, comb. nov.

Basionym: Stipa feresetacea Vickery, S.W.L. Jacobs \& J. Everett, Telopea 3: 58 (1986).

Austrostipa flavescens (Labill.) S.W.L. Jacobs $\mathcal{E}$ J. Everett, comb. nov.

Basionym: Stipa flavescens Labillardiäre, Nov. Holl. Pl. 1: 24, pl. 30 (1804); Vickery, Jacobs \& Everett (1986: 59-61).

Austrostipa geoffreyi S.W.L. Jacobs $\mathcal{E}$ J. Everett, sp. nov. A. juncifoliae affinis, sed habitu valde robustiore, vaginis folii latioribus, foliis longioribus, inflorescentia densiore, arista ad $7 \mathrm{~cm}$ longa, omnibus partibus floralibus grandioribus, differt.

Holotype: Western Australia: Lake King, 3305"26'S 119³3"37'E, S.W.L. Jacobs 7030, 2.12.1993. Gypsaceous islands connected by causeway. Islands with samphires and. Frankenia. (NSW 293105; duplicate PERTH).

Caespitose perennial to 1.8 metres tall, with short rhizomes, without a basal tuft of leaves. Culms erect, terete, $2-4 \mathrm{~mm}$ wide near the base, + compressible, ribbed about the nodes, glabrous; nodes c. 3 , exserted, to twice the width of adjacent internodes. Leaf sheaths loose, moderately ribbed; basal sheath $8-14 \mathrm{~mm}$ wide, shortly puberulous with hairs $<0.3 \mathrm{~mm}$ long between the ribs; upper sheath $5-10 \mathrm{~mm}$ wide, shortly puberulous between the ribs; margins glabrous. Ligule membranous, acute, 6-12 $\mathrm{mm}$ long, shortly puberulous with hairs $<0.1 \mathrm{~mm}$ long. Leaf blade rolled, 1-2 $\mathrm{mm}$ in diameter, to 1 metre long; abaxial surface smooth and glabrous; adaxial surface 
densely scaberulous with minute siliceous prickles; margins glabrous. Panicle $30-40$ $\mathrm{cm}$ long, exserted, with fascicles of unequal, few-flowered compound branches, barely spreading, 3-5 cm wide (excluding awns); axis terete, glabrous; branches to $12 \mathrm{~cm}$ long, slightly flattened, the lower $1-2 \mathrm{~cm}$ shortly puberulous on the adaxial surface; pedicels 3-13 mm long, terete, glabrous. Spikelets 10-16 mm long (excluding awn). Glumes subequal, acute to acuminate, straw-coloured; lower glume 12-15 mm long, lower 50\% 3-nerved; upper glume 12-16 mm long, lower 60\% 3-nerved. Floret cylindrical, without a neck, 9-12 mm long (including callus). Lemma smooth, sericeous with white hairs 1-1.5 mm long; lobes $2.5 \mathrm{~mm}$ long; coma $3 \mathrm{~mm}$ long. Callus 2-3 mm long, weakly bent at the tip; sericeous with white hairs $0.75 \mathrm{~mm}$ long. Awn 5-8 cm long, twice bent, $0.25-0.3 \mathrm{~mm}$ wide near the base; column 10-20 $\mathrm{mm}$ long, $5-10 \mathrm{~mm}$ to the first bend, scabrous with hairs $0.1-0.2 \mathrm{~mm}$ long; bristle darker than the column, scabrous with hairs $0.1-0.15 \mathrm{~mm}$ long. Palea equal to the lemma, 3(-4)-toothed, slightly depressed between the nerves, surface smooth, sericeous along the centre back with hairs $0.5-1.5 \mathrm{~mm}$ long, margins glabrous. Lodicules 2, abaxial, membranous, obtuse, 1-2 mm long. Anthers not seen. Immature caryopsis $6 \mathrm{~mm}$ long, hilum $75 \%$ the length, embryo $50 \%$ the length.

Similar to Stipa juncifolia but differs in being considerably more robust with wider sheaths, longer leaves, a denser inflorescence, an awn to $7 \mathrm{~cm}$ long, and larger in its floral parts.

This species is named after Geoffrey Thomas Jacobs, son of SWLJ, who has helped on many field trips and was present when the Type was collected.

Distribution: Only known from the islands of, and the causeway across, Lake King, a salt lake in southern Western Australia.

Specimens examined: Western Australia: Roe: Lake King, George 10466, 11.11.1970 (PERTH, NSW); Jacobs 5854 \& P. Wilson, 11.6.1988 (NSW, UTC).

Vickery et al. (1986) noted that a specimen of Austrostipa juncifolia (as Stipa juncifolia) from Lake King was considerably more robust than others of the same species even from the same locality. We have since been able to study both taxa in the field and under cultivation. At Lake King, Austrostipa juncifolia grows around the lake behind the highest strand line; the more robust Austrostipa geoffreyi grows on the islands and along the causeway across the lake - they do not appear to grow as mixed populations. Under cultivation, all of the observed distinctions between the two taxa are maintained, with the additional observation that $A$. geoffreyi is much more difficult to maintain in cultivation than $A$. juncifolia. After collecting more material and examining the taxa in the field and under cultivation we consider that A. geoffreyi warrants recognition at the species level.

Austrostipa gibbosa (Vickery) S.W.L. Jacobs \& J. Everett, comb. nov.

Basionym: Stipa gibbosa Vickery, Telopea 2: 14 (1980); Vickery, Jacobs \& Everett (1986: 62-63).

Austrostipa hemipogon (Benth.) S.W.L. Jacobs \& J. Everett, comb. nov.

Basionym: Stipa hemipogon Bentham, Fl. Austral. 7: 569 (1878); Vickery, Jacobs \& Everett (1986: 63-65).

Austrostipa juncifolia (Hughes) S.W.L. Jacobs \& J. Everett, comb. nov.

Basionym: Stipa juncifolia Hughes, Kew Bull. 1921: 11 (1921); Vickery, Jacobs \& Everett (1986: 66-67).

Austrostipa lanata (Vickery, S.W.L. Jacobs \& J. Everett) S.W.L. Jacobs \& J. Everett, comb. nov.

Basionym: Stipa lanata Vickery, S.W.L. Jacobs \& J. Everett, Telopea 3: 68-69 (1986). 
Austrostipa macalpinei (Reader) S.W.L. Jacobs \& J. Everett, comb. nov.

Basionym: Stipa macalpinei Reader, Victorian Naturalist 15: 143 (1899); Vickery, Jacobs \& Everett (1986: 70-71).

Austrostipa metatoris (J. Everett \& S.W.L. Jacobs) S.W.L. Jacobs \& J. Everett, comb. nov. Basionym: Stipa metatoris J. Everett \& S.W.L. Jacobs, Telopea 2: 399 (1983); Vickery, Jacobs \& Everett (1986: 73-74).

Austrostipa mollis (R. Br.) S.W.L. Jacobs \& J. Everett, comb. nov.

Basionym: Stipa mollis R. Brown, Prodr.: 174 (1810); Vickery, Jacobs \& Everett (1986: 74-75).

Austrostipa muelleri (Tate) S.W.L. Jacobs \& J. Everett, comb. nov.

Basionym: Stipa muelleri Tate, Trans. \& Proc. Roy. Soc. South Austr. 7: 70 (1885); Vickery, Jacobs \& Everett (1986: 77-78).

Austrostipa multispiculis (J.M. Black) S.W.L. Jacobs \& J. Everett, comb. nov.

Basionym: Stipa multispiculis J.M. Black, Trans. \& Proc. Roy. Soc. South Austr. 65: 333 (1941); Vickery, Jacobs \& Everett (1986: 78-79).

Austrostipa mundula (J.M. Black) S.W.L. Jacobs \& J. Everett, comb. nov.

Basionym: Stipa mundula J.M. Black, Trans. \& Proc. Roy. Soc. South Austr. 65: 333 (1941); Vickery, Jacobs \& Everett (1986: 79-80).

Austrostipa nitida (Summerhayes \& C.E. Hubb.) S.W.L. Jacobs \& J. Everett, comb. nov. Basionym: Stipa nitida Summerhayes \& C.E. Hubbard, Kew Bull. 1927: 80 (1927); Vickery, Jacobs \& Everett (1986: 82-84).

Austrostipa nivicola (J.H. Willis) S.W.L. Jacobs \& J. Everett, comb. nov.

Basionym: Stipa nivicola J.H. Willis, Victorian Naturalist 73: 149 (1957); Vickery, Jacobs \& Everett (1986: 85-86).

Austrostipa nodosa (S.T. Blake) S.W.L. Jacobs \& J. Everett, comb. nov.

Basionym: Stipa nodosa S.T. Blake, Proc. Roy. Soc. Queensland 62: 89 (1952); Vickery, Jacobs \& Everett (1986: 86-87).

Austrostipa nullarborensis (Vickery, S.W.L. Jacobs \& J. Everett) S.W.L. Jacobs \& J. Everett, comb. nov.

Basionym: Stipa nullarborensis Vickery, S.W.L. Jacobs \& J. Everett, Telopea 3: 88-89 (1986).

Austrostipa nullanulla (J. Everett \& S.W.L. Jacobs) S.W.L. Jacobs \& J. Everett, comb. nov. Basionym: Stipa nullanulla J. Everett \& S.W.L. Jacobs, Telopea 2: 398 (1983); Vickery, Jacobs \& Everett (1986: 89-90).

Austrostipa oligostachya (Hughes) S.W.L. Jacobs $\mathcal{E} J$. Everett, comb. nov.

Basionym: Stipa oligostachya Hughes, Kew Bull. 1921: 12 (1921); Vickery, Jacobs \& Everett (1986: 90-91).

Austrostipa petraea (Vickery) S.W.L. Jacobs \& J. Everett, comb. nov.

Basionym: Stipa petraea Vickery, Telopea 2: 15 (1980); Vickery, Jacobs \& Everett (1986: 91-92).

Austrostipa pilata (S.W.L Jacobs \& J. Everett) S.W.L. Jacobs $\mathcal{E}$ J. Everett, comb. nov. Basionym: Stipa pilata S.W.L. Jacobs \& J. Everett in Vickery, S.W.L. Jacobs \& J. Everett, Telopea 3: 92-93 (1986).

Austrostipa platychaeta (Hughes) S.W.L. Jacobs \& J. Everett, comb. nov.

Basionym: Stipa platychaeta Hughes, Kew Bull. 1921: 16 (1921); Vickery, Jacobs \& Everett (1986: 93-94). 
Austrostipa plumigera (Hughes) S.W.L. Jacobs \& J. Everett, comb. nov.

Basionym: Stipa plumigera Hughes, Kew Bull. 1921: 20 (1921); Vickery, Jacobs \& Everett (1986: 94-95).

Austrostipa puberula (Steud.) S.W.L. Jacobs \& J. Everett, comb. nov.

Basionym: Stipa puberula Steudel, Syn. Pl. Glum. 1: 128 (1854); Vickery, Jacobs \& Everett (1986: 95-96).

Austrostipa pubescens (R. Br.) S.W.L. Jacobs \& J. Everett, comb. nov.

Basionym: Stipa pubescens R. Brown, Prodr.: 174 (1810); Vickery, Jacobs \& Everett (1986: 97-98).

Austrostipa pubinodis (Trin. \& Rupr.) S.W.L. Jacobs \& J. Everett, comb. nov.

Basionym: Stipa pubinodis Trinius \& Ruprecht, Mém. Acad. Imp. Sci. St Pétersbourg, Ser. 6, Sci. Math., Seconde Pt Sci. Nat. 5: 50 (1842); Vickery, Jacobs \& Everett (1986: 99-100).

Austrostipa pycnostachya (Benth.) S.W.L. Jacobs \& J. Everett, comb. nov.

Basionym: Stipa pycnostachya Bentham, Fl. Austr. 7: 568 (1878); Vickery, Jacobs \& Everett (1986: 100-101).

Austrostipa ramosissima (Trin.) S.W.L. Jacobs \& J. Everett, comb. nov.

Basionym: Urachne ramosissima Trinius, Gram. Unifl.: 173 (1824).

Synonym: Stipa ramosissima (Trinius) Trinius (1830: 74); Vickery, Jacobs \& Everett (1986: 101-103).

Austrostipa rudis (Spreng.) S.W.L. Jacobs \& J. Everett, comb. nov.

Basionym: Stipa rudis Sprengel, Syst. Veg. Cur. Post. 4: 31 (1827); Vickery, Jacobs \& Everett (1986: 103-106).

Austrostipa rudis subspecies rudis (autonym)

Synonyms: Stipa nervosa var. neutralis Vickery (1951: 335).

Stipa rudis subspecies rudis (Everett \& Jacobs 1983: 396).

Austrostipa rudis subspecies australis (J. Everett \& S.W.L. Jacobs) S.W.L. Jacobs $\mathcal{E} J$. Everett, comb. nov.

Basionym: Stipa rudis subspecies australis J. Everett \& S.W.L. Jacobs, Telopea 2: 396 (1983); Vickery, Jacobs \& Everett (1986: 105-106).

Austrostipa rudis subspecies nervosa (Vickery) S.W.L. Jacobs \& J. Everett, comb. nov. Basionym: Stipa nervosa var. nervosa Vickery, Contrib. N.S.W. Natl. Herb. 1: 335 (1951). Synonym: Stipa rudis subspecies nervosa (Vickery) J. Everett \& S.W.L. Jacobs (1983: 396); Vickery, Jacobs \& Everett (1986: 106).

Austrostipa scabra (Lindl.) S.W.L. Jacobs \& J. Everett, comb. nov.

Basionym: Stipa scabra Lindley in Mitchell, Journ. Trop. Austr.: 31 (1848); Vickery, Jacobs \& Everett (1986: 107-111).

Austrostipa scabra subspecies scabra (autonym)

Synonym: Stipa scabra subspecies scabra (Vickery, Jacobs \& Everett 1986: 108-109).

Austrostipa scabra subspecies falcata (Hughes) S. W.L. Jacobs \& J. Everett, comb. nov. Basionym: Stipa falcata Hughes, Kew Bull. 1921: 14 (1921).

Synonym: Stipa scabra subspecies falcata (Hughes) Vickery, S.W.L. Jacobs \& J. Everett (1986: 110-111).

Austrostipa semibarbata (R. Br.) S.W.L. Jacobs \& J. Everett, comb. nov.

Basionym: Stipa semibarbata R. Brown, Prodr.: 174 (1810); Vickery, Jacobs \& Everett (1986: 111-113). 
Austrostipa setacea (R. Br.) S.W.L. Jacobs \& J. Everett, comb. nov.

Basionym: Stipa setacea R. Brown, Prodr.: 174 (1810); Vickery, Jacobs \& Everett (1986: 113-115).

Austrostipa stipoides (Hook. f.) S.W.L. Jacobs $\mathcal{E}$ J. Everett, comb. nov.

Basionym: Dichelachne stipoides Hooker f., Fl. Nov.-Zel. 1: 294, t. 66 (1853).

Synonym: Stipa stipoides (Hook. f.) Veldkamp (1974: 11); Vickery, Jacobs \& Everett (1986: 116-117).

Austrostipa stuposa (Hughes) S.W.L. Jacobs \& J. Everett, comb. nov.

Basionym: Stipa stuposa Hughes, Kew Bull. 1921: 20 (1921); Vickery, Jacobs \& Everett (1986: 117-118).

Austrostipa tenuifolia (Steud.) S.W.L. Jacobs \& J. Everett, comb. nov.

Basionym: Stipa tenuifolia Steudel, Syn. Pl. Glum. 1: 128 (1854); Vickery, Jacobs \& Everett (1986: 119-120).

Austrostipa trichophylla (Benth.) S.W.L. Jacobs \& J. Everett, comb. nov.

Basionym: Stipa trichophylla Bentham, Fl. Austr. 7: 570 (1878); Vickery, Jacobs \& Everett (1986: 121-122).

Austrostipa tuckeri (F. Muell.) S.W.L. Jacobs \& J. Everett, comb. nov.

Basionym: Stipa tuckeri F. Mueller, Fragm. 9: 129 (1881); Vickery, Jacobs \& Everett (1986: 123).

Austrostipa variabilis (Hughes) S.W.L. Jacobs \& J. Everett, comb. nov.

Basionym: Stipa variabilis Hughes, Kew Bull. 1921: 15 (1921); Vickery, Jacobs \& Everett (1986: 124-125).

Austrostipa velutina (Vickery, S.W.L. Jacobs \& J. Everett) S.W.L. Jacobs \& J. Everett, comb. nov.

Basionym: Stipa velutina Vickery, S.W.L. Jacobs \& J. Everett, Telopea 3: 126 (1986).

Austrostipa verticillata (Nees ex Spreng.) S.W.L. Jacobs \& J. Everett, comb. nov.

Basionym: Stipa verticillata Nees ex Sprengel, Syst. Veg. Cur. Post. 4: 30 (1827); Vickery, Jacobs \& Everett (1986: 127-128).

Austrostipa vickeryana (J. Everett \& S.W.L. Jacobs) S.W.L. Jacobs \& J. Everett, comb. nov. Basionym: Stipa vickeryana J. Everett \& S.W.L. Jacobs, Telopea 2: 397 (1983); Vickery, Jacobs \& Everett (1986: 128-129).

Austrostipa wakoolica (Vickery, S.W.L. Jacobs \& J. Everett) S.W.L. Jacobs \& J. Everett, comb. nov.

Basionym: Stipa wakoolica Vickery, S.W.L. Jacobs \& J. Everett, Telopea 3: 129-130 (1986).

\section{Key to the subgenera}

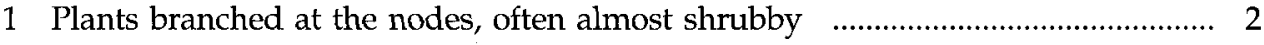

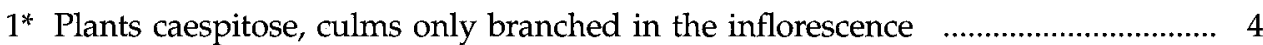

2 The whole panicle acting as the diaspore, wind-dispersed; panicle branches plumose subgen. Petaurista

$2^{*}$ The floret acting as the diaspore; panicle branches scabrous or glabrous 3

3 Culms with simple branches; spikelets mostly $>4 \mathrm{~mm}$ long 
$3^{*}$ Culms with whorled branches; spikelets $<4 \mathrm{~mm}$ long subgen. Bambusina

4 Lemma glabrous for varying distances below the apex 5

$4^{*}$ Lemma hairy all over, though hairs may be of different lengths

5 The lemma glabrous or almost so, the surface shining

subgen. Lanterna

$5^{*}$ Lemma glabrous only in the top half, the glabrous portion rough, tuberculate or 'crystalline' (with individual small bumps or ridges of translucent silica)

subgen. Tuberculatae

6 Lemma with 2 membranous lobes usually $>1 \mathrm{~mm}$ long

subgen. Lobatae

$6^{*}$ Lemma with $0-1$ lobes or, if 2 present, then $<1 \mathrm{~mm}$ long

7 Bristle of the awn falcate; lemma narrow, often evenly covered with comparatively sparse, short, white hairs subgen. Falcatae

$7^{*}$ Bristle usually straight or only slightly curved, if falcate then the lemma not evenly covered with comparatively sparse, short, white hairs

8 Column of the awn plumose or long-pubescent; lemma never covered with brown hairs

subgen. Austrostipa

$8^{*}$ Column of the awn scabrous or glabrous, if plumose then the mature lemma covered with brown hairs

9 Palea with an adaxial groove, the lemma margins fitting into the groove and not overlapping subgen. Aulax

9* Palea entire; lemma margins overlapping 10

10 Glumes broad and inflated around the floret; lemma with a coma and a short callus at an angle to the long axis of the floret; panicle usually open and spreading

subgen. Ceres

$10^{*}$ Glumes narrow, close around the floret; lemma usually with a coma and a long, fine, straight callus aligned with the long axis of the floret; panicle often contracted with short branches

11 Plants perennial but with very short and narrow leaves in a small tussock, inflorescence many times longer than these small basal leaves (often missing from specimens) and often mistaken as being from annual plants; awns usually $>7 \mathrm{~cm}$ long

subgen. Longiaristatae

11* Plants perennial and caespitose, the inflorescence never more than 2-3 times as long as the basal leaves (unless grazed); awns usually $<7 \mathrm{~cm}$ long

12 Lemma hairs often becoming dark reddish brown at maturity; sometimes a patch of shorter hairs present below the apex; awn comparatively long; more commonly plants of inland red sandy or rocky soils

subgen. Eremophilae

$12^{*}$ Lemma hairs unchanging or becoming yellow at maturity, but never dark reddish brown; patches of shorter hairs never present; awn comparatively short; more commonly plants near the coast or on light-coloured sandy soils 


\section{Austrostipa subgenus Austrostipa (autonym)}

A. mollis (Type), A. hemipogon, A. densiflora, A. campylachne, A. stuposa, A. semibarbata, A. aquarii.

Column of the awn plumose or long-pubescent. Lemma usually without a coma.

Austrostipa subgenus Arbuscula S.W.L. Jacobs \& J. Everett, subgen.nov.

A. acrociliata (Type), A. breviglumis, A. nullarborensis, A. platychaeta, A. pycnostachya.

Culmi plerumque ramosi sed ramis non verticillatis. Ligula folii longa. Paniculae grandes, sparsae, effusae.

Culms usually branched though the branches not whorled. Leaves with long ligules. Panicles large, sparse and spreading.

The epithet is Latin for small tree or shrub in reference to the shrubby growth form of these species.

Austrostipa subgenus Longiaristatae S.W.L. Jacobs \& J. Everett, subgen. nov.

A. compressa (Type), A. macalpinei.

Caespites minutos perennesque formans, culmis florentibus grandis quotannis praesentibus. Lemma pallidum, arista longa paene recta.

Forming minute perennial tufts with large flowering culms produced annually. Lemma pale with a long, almost straight awn.

The epithet refers to the long awns characteristic of these species.

Austrostipa subgenus Aulax S.W.L. Jacobs \& J. Everett, subgen. nov.

A. setacea (Type), A. feresetacea.

Palea adaxialiter profunde sulcata. Ligula folii longa (ad $2 \mathrm{~mm}$ ).

Palea with a deep adaxial groove. Ligule long ( $>2 \mathrm{~mm}$ ).

The epithet is Latin for 'furrow' or 'groove', referring to the adaxial groove on the palea.

Austrostipa subgenus Lanterna S.W.L. Jacobs \& J. Everett, subgen. nov.

A. nullanulla (Type), A. lanata, A. vickeryana.

Lemma superficie nitenti, glabra vel subglabra.

Lemma with a shiny surface, glabrous or almost so.

The epithet is from Latin 'lanterna', a lantern or lamp, referring to the bright, shining surface of the lemma.

Austrostipa subgenus Lancea S.W.L. Jacobs \& J. Everett, subgen. nov.

A. flavescens (Type), A. exilis, A. mundula, A. echinata, A. velutina, A. multispiculis, A. crinita.

Glumae angustae, circa flosculum confertae. Coma lemmatis praesens; callus lemmatis longus, gracilis rectusque. Panicula saepe contracta, ramis brevibus.

Glumes narrow, close around the floret. Lemma with a coma and a long, fine and straight callus. Panicle often contracted, with short branches.The epithet is Latin for 'small lance', referring to the shape of the floret. 
Austrostipa subgenus Lobatae S.W.L. Jacobs \& J. Everett, subgen. nov.

A. juncifolia (Type), A. geoffreyi, A. stipoides, A. petraea.

Lemma ad apicem lobis longis acutisque.

Lemma with long, acute lobes at the apex. The epithet refers to the long lobes at the lemma apex characterising these species.

Austrostipa subgenus Tuberculatae S.W.L. Jacobs \& J. Everett, subgen. nov.

A. rudis (Type), A. aphylla, A. oligostachya, A. pubescens, A. pubinodis, A. nivicola, A. muelleri.

Lemma versus apicem plusminusve glabrum, superficie scabra vel tuberculata. Glumae valde costatae.

Lemma glabrous for varying lengths below the apex, the surface rough ('crystalline' - with clear small lumps or ridges of translucent silica - or tuberculate). Glumes strongly ribbed.

The epithet refers to the tuberculate surface of the upper lemma characteristic of this group of species.

Austrostipa subgenus Falcatae S.W.L. Jacobs \& J. Everett, subgen. nov.

A. nitida (Type), A. nodosa, A. scabra, A. variabilis, A. trichophylla, A. blakei, A. drummondii, A. tenuifolia, A. pilata.

Arista falcata. Lemma angustum aciculareque.

Bristle of awn falcate. Lemma narrow and needle-like.

The epithet has been used informally for a long time (e.g. Hughes 1922) and refers to the sickle-shaped bristle of the awn.

Austrostipa subgenus Ceres S.W.L. Jacobs \& J. Everett, subgen. nov.

A. gibbosa (Type), A. aristiglumis, A. curticoma, A. bigeniculata, A. blackii, A. dongicola.

Glumae latae, circum flosculum dilatatae. Coma lemmatis praesens; callus lemmatis valde angulatus brevisque. Panicula effusa, ramis longis.

Glumes broad and inflated around the floret. Lemma with a coma and a short, strongly angled callus. Panicle expanded with long branches.

The epithet is a name for the Roman goddess of grain and agriculture and refers to both the almost Oat-like appearance of the broad glumes and the high ranking many of the species in this group are given as pasture species.

Austrostipa subgenus Eremophilae S.W.L. Jacobs \& J. Everett, subgen. nov.

A. eremophila (Type), A. metatoris, A. wakoolica, A. puberula, A. plumigera, A. centralis.Coma lemmatis praesens; pili lemmatis ad maturitatem saepe fuscescentes. Arista longa.

Lemmas with a coma. The lemma hairs often darkening at maturity. Awn long.

The epithet is derived from the name of one of the species which in turn is Latinised Greek usually translated as being desert- or wilderness-loving.

Austrostipa subgenus Petaurista S.W.L. Jacobs \& J. Everett, subgen. nov.

A. elegantissima (Type), A. tuckeri.

Panicula grandis pyramidalisque vice diasporae fungens. Culmi ramosi sed ramis non verticillatis. Lemma palea minimum 2-plo longius.

Panicle large and pyramidal, acting as the diaspore. Panicle branches plumose. Culms branched but the branches not whorled. Palea less than half the length of the lemma.

The epithet is Latin for 'tumbler' or 'vaulter', referring to the wind-dispersed inflorescence. 
Austrostipa subgenus Bambusina S.W.L. Jacobs \& J. Everett, subgen. nov.

A. verticillata (Type), A. ramosissima.Culmi ramis verticillatis. Flosculi parvi (minus quam $4 \mathrm{~mm}$ ).

Culms with whorled branches. Florets small ( $<4 \mathrm{~mm}$ long).

The epithet is a Latinised diminutive of the Malay word 'bambu', referring to the resemblance of plants of the two species to small clumps of bamboo.

\section{Nassella}

Nassella Desvaux in Gay (1854: 263); Barkworth (1990, 1993).

Nassella hyalina (Nees) Barkworth (1990: 610).

Basionym: Stipa hyalina Nees (1829: 378); Vickery, Jacobs \& Everett (1986: 66).

Nassella leucotricha (Trin. \& Rupr.) Pohl in Barkworth (1990: 610). Barkworth (pers. comm.) states that this should be cited as 'Pohl in Barkworth' even though she mistakenly stated on p. 608 that only two new combinations were being made by Pohl instead of the three made.

Basionym: Stipa leucotricha Trinius \& Ruprecht (1842: 54); Vickery, Jacobs \& Everett (1986: 69-70).

Nassella megapotamia (Spreng. ex Trin.) Barkworth (1990: 610-611).

Basionym: Stipa megapotamia Spreng. ex Trinius (1830: 77); Vickery, Jacobs \& Everett (1986: 72-73).

Nassella neesiana (Trin. E Rupr.) Barkworth (1990: 611).

Basionym: Stipa neesiana Trinius \& Ruprecht (1842: 27); Vickery, Jacobs \& Everett (1986: 81).

Nassella trichotoma (Nees) Hack. ex Arechavaleta (1895: 276).

Basionym: Stipa trichotoma Nees (1829: 375); Vickery \& Jacobs (1980: 21-23).

\section{Acknowledgements}

We would like to thank Karen Wilson for discussion and the Latin diagnoses, Elizabeth Brown, Henry Connor and Elizabeth Edgar for their helpful and constructive comments on the manuscript, Elizabeth Edgar for obtaining some critical material for us at short notice, and both Mary Barkworth and Henry Connor for helpful lengthy discussions on Stipoid grasses over the years.

\section{References}

Arechavaleta, B.J. y (1895) Las Gramineas Uruguayas. Annales del Museo nacional de Montevideo 1(3): 213-292.

Barkworth, M.E. (1990) Nassella (Gramineae, Stipeae): revised interpretation and nomenclatural changes. Taxon 39: 597-614.

Barkworth, M.E. (1993) North American Stipeae (Gramineae): taxonomic changes and other comments. Phytologia 74: 1-25.

Barkworth, M.E. \& Everett, J. (1986) Evolution in the Stipeae: identification and relationships of its monophyletic taxa. PP251-264 in : Soderstrom, T.R., Hilu, K.W., Campbell, C.S. \& Barkworth, M.E. (eds), Grass systematics and evolution (Smithsonian Institution Press: Washington).

Beauvois, P. de (1812) Essai d'une nouvelle agrostographie (Fain: Paris).

Bentham, G. (1870) Flora Australiensis, vol. 7 (L. Reeve \& Co.: London).

Black, J.M. (1941) Additions to the flora of South Australia. Transactions and Proceedings of the Royal Society of South Australia 65: 333-334. 
Brown, R. (1810) Prodromus florae Novae Hollandiae (J. Johnson \& Co.: London).

Buchanan, J. (1880) Manual of the indigenous grasses of New Zealand addenda (James Hughes: Wellington).

Clayton, W.D. \& Renvoize, S.A. (1986) Genera Graminum (Her Majesty's Stationery Office: London).

Clifford, H.T. \& Watson, L. (1977) Identifying grasses (University of Queensland Press: Brisbane).

Everett, J. (1990) Systematic relationships of the Australian Stipeae (Poaceae). Unpublished MSc. thesis, University of Sydney.

Everett, J. \& Jacobs, S.W.L. (1983) Studies in Australian Stipa (Poaceae). Telopea 2: 391-400.

Freycinet, H.L.C. de S. de (1830) Voyage autour du monde ... sur les corvettes de S.M. l'Uranie et la Physicienne. Botanique (Pillet-ainé: Paris).

Gay, C. (1854) Historia fiscia y politica de Chile ... Botanica. vol. 6. (Museo historia natural: Santiago).

Hooker, J.D. (1853) Flora Novae-zelandiae, vol. 1. (L. Reeve \& Co.: London).

Hsiao, C., Chatterton, N.J., Assay, K.H. \& Jensen, K.B. (1995) Molecular phylogeny of the Pooideae (Poaceae) based on nuclear rDNA (ITS) sequences. Theoretical and Applied Genetics 90: 389-398.

Hubbard, C.E. (1925) in: Decades Kewensis: plantarum novarum in herbario horti regii conservatorum. Decas CXIII. Kew Bulletin 1925: 426-433.

Hughes, D.K. (1921) A revision of the Australian species of Stipa. Kew Bulletin 1921: 1-30.

Hughes, D.K. (1922) Further notes on the Australian species of Stipa. Kew Bulletin 1922: 15-22.

Jacobs, S.W.L. \& Everett, J. (1993) Piptatherum, Nassella and Stipa. Pp 637-650 in Harden, G.J. (ed.) Flora of New South Wales vol. 4. (University of New South Wales Press: Sydney).

Jacobs, S.W.L., Everett, J. and Barkworth, M.E. (1995) Clarification of morphological terms used in the Stipeae (Gramineae), and a reassessment of Nassella in Australia. Taxon 44: 33-41.

Jacobs, S.W.L., Everett, J. Connor, H.E., \& Edgar, E. (1989) Stipoid grasses in New Zealand. New Zealand Journal of Botany 27: 569-582.

Labillardiäre, J.J.H. de (1804) Novae Hollandiae plantarum specimen, vol. 1. (Dominae Huzard: Paris).

Lehmann, J.G.C. (1846) Plantae Preissianeae, vol. 2. (Sumptibus Meissneri: Hamburg).

Mitchell, T.L. (1848) Journal of an expedition into the interior of Tropical Australia in search of a route from Sydney to the Gulf of Carpentaria (Longman, Brown, Green \& Longmans: London).

Mueller, F. (1881) Fragmenta Phytographie Australiae 11: 128-129.

Mueller, F. (1855) Description of fifty new Australian plants, chiefly from the colony of Victoria. Transactions and Proceedings of the Victorian Institute for the Advancement of Science for 185455 [1:] 28-54.

Nees von Esenbeck C.G.D. (1829) Agrostologia brasiliensis (J.C. Cottae: Stuttgart \& Turbingen).

Niles, C. \& Chase, A. (1925) A bibliographic study of Beauvois' Agrostographie. Contributions from the United States National Herbarium 24: 135-214.

Reader, F.M. (1897) Contributions to the flora of Victoria no. II. Victorian Naturalist 13: 167-168.

Reader, F.M. (1899) Contributions to the flora of Victoria no. VIII. Victorian Naturalist 15: 143-145.

Reader, F.M. (1901) Contributions to the flora of Victoria no. X. Victorian Naturalist 17: 154-155.

Reeder, J.R. (1957) The embryo in grass systematics. American Journal of Botany 44: 756-768.

Rodway, L. (1903) The Tasmanian Flora (John Vail, Government Printer: Hobart).

Sprengel, K.P.J. (1827) Systema Vegetabilum, vol. 4. Curae Posteriores ad vol. 1. (Librariae Dieterichianeae: Gîttingen).

Steudel, E.G. (1854) Synopsis plantarum glumacearum, vol. 1. (J.B. Metzler: Stuttgart).

Summerhayes, V.S. \& Hubbard, C.E. (1927) in: Decades Kewensis: plantarum novarum in herbario horti regii consevatorum. Kew Bulletin 1927: 75-81.

Swofford, D.L. (1985). PAUP: phylogenetic analysis using parsimony. Version 2.4. Illinois Natural History Survey, Campaign, Illinois.

Tate, R. (1885) Descriptions of new species of South Australian plants. Transactions and Proceedings of the Royal Society of South Australia 7: 67-71.

Townrow, J.E.S. (1970) The genus Stipa L. in Tasmania. Part 1 - introduction and identification. Papers and Proceedings of the Royal Society of Tasmania 104: 81-98.

Trinius, C.B. (1824) De Graminibus unifloris et sesquifloris (Impensis Acadamiae Imperialis Scientarum: Saint Petersburg).

Trinius, C.B. (1830) Graminum genera. Mémoires de l'Académie Imperialis Scientiarum SaintPétersbourg Séries 6, Science et Math 1: 54-93. 
Trinius, C.B. \& Ruprecht, FJ. (1842) Species graminum stipaceorum. A preprint from: (1843) Mémoires de l'Académie Imperialis Scientiarum Saint-Pétersbourg Séries 6, Science et Math 7: 1-189.

Veldkamp, J.F. (1974) A taxonomic revision of Dichelachne Endl. (Gramineae) with some new combinations in Stipa L. and Oryzopsis Michx. Blumea 22: 5-12.

Vickery, J.W. (1951) Contributions to the taxonomy of Australian grasses. Contributions from the New South Wales National Herbarium 1: 322-343.

Vickery, J.W. (1980) Four new species of Stipa (Poaceae). Telopea 2: 11-15.

Vickery, J.W. \& Jacobs, S.W.L. (1980) Nassella and Oryzopsis (Poaceae) in New South Wales. Telopea 2: $17-23$.

Vickery, J.W., Jacobs, S.W.L. \& Everett, J. (1986) Taxonomic studies in Stipa (Poaceae) in Australia. Telopea 3: 1-132.

Walsh, N.G. (1994) Poaceae. Pp 356-627 in Walsh, N.G. \& Entwistle, T.J. (eds) Fora of Victoria vol. 2. (Inkata Press: Melbourne).

Willis, J.H. (1957) Vascular flora of Victoria and South Australia. Victorian Naturalist 73: 149-160.

Manuscript received 10 August 1995

Manuscript accepted 12 February 1996 